\title{
Scalable Privacy-Preserving Data Mining with Asynchronously Partitioned Datasets
}

\author{
Hiroaki Kikuchi ${ }^{1}$, Daisuke Kagawa ${ }^{1}$, Anirban Basu ${ }^{1}$, Kazuhiko Ishii ${ }^{2}$, \\ Masayuki Terada ${ }^{2}$, and Sadayuki Hongo ${ }^{3}$ \\ 1 Graduate School of Engineering, Tokai University, \\ 1117, Kitakaname, Hiratsuka, Kanagawa, 259-1292, Japan \\ kikn@tokai.ac.jp, \{nagomin03, abasu\}@cs.dm.u-tokai.ac.jp \\ 2 NTT DoCoMo Inc. \\ 3-5 Hikarinooka, Yokosuka-shi, Kanagawa, 239-8536, Japan \\ \{ishiikaz, teradama\}@nttdocomo.co.jp \\ 3 Department of Frontier Information Engineering, \\ Faculty of Advanced Engineering, Hokkaido Institute of Technology 7-15 Maeda, \\ Teine-ku, Sapporo, Hokkaido, 006-8585, Japan \\ hongo@it.ac.jp
}

\begin{abstract}
In the Naïve Bayes classification problem using a vertically partitioned dataset, the conventional scheme to preserve privacy of each partition uses a secure scalar product and is based on the assumption that the data is synchronised amongst common unique identities. In this paper, we attempt to discard this assumption in order to develop a more efficient and secure scheme to perform classification with minimal disclosure of private data. Our proposed scheme is based on the work by Vaidya and Clifton[1], which uses commutative encryption to perform secure set intersection so that the parties with access to the individual partitions have no knowledge of the intersection. The evaluations presented in this paper are based on experimental results, which show that our proposed protocol scales well with large sparse datasets.
\end{abstract}

\section{Introduction}

Privacy-preserving data mining aims to allow computation of useful aggregate statistics over the entire dataset without compromising the privacy of individual data. The parties collaborating to obtain aggregate results may not fully trust each other, such as a Sybil attack 2] resistant recommendation system [3] or the Naïve Bayes classifier [1]. Such parties may also be competitors in the same field, for example companies which may have privacy policies restricting access to each other's customer datasets.

Vertically partitioned data is an important data distribution model often found in real life. For example, Table 1 illustrates two datasets partitioned vertically where attributes $A_{1}$ and $A_{2}$ are owned by Alice $(A)$; and $\operatorname{Bob}(B) 1$ owns the attribute $A_{3}$ and a target class $C$, which indicates whether or not to play tennis on

\footnotetext{
${ }^{1}$ From this point forward, we use Alice, $A$, and party $A$ interchangeably; and the same for Bob, $B$ or party $B$.
} 
the day. Alice and Bob separately collect the different features, e.g. temperature, humidity, etc. for each day. Collaboratively performing Naïve Bayes classification allows them to accurately predict the decision to play or not, i.e. predict $C$ given $A_{1}, A_{2}$ and $A_{3}$, although they can not share each other's datasets.

Syncronous and Asynchronous Partitions: Vaidya and Clifton presented, in [1, a secure protocol for Naïve Bayes classification for vertically partitioned datasets without revealing the individual partitions. Their protocol combines homomorphic public-key encryption algorithm to compute scalar product of two vectors, with the secure function evaluation [4] for comparison of class $c \in C$ in terms of conditional attributes, i.e. $\operatorname{Pr}\left(C=c \mid a_{1}, a_{3}\right)$.

Their protocol assumes that the input vectors are of the same dimensions. Hence, the partitioned datasets are synchronous with the days (in our example in Table 10 when the attributes are observed. However, datasets may not always be synchronous. For example, the dataset in Table 2 is vertically but asynchronously partitioned, where attributes are stored with common IDs. This type of asynchronous partitions are of frequent occurrences in our daily lives. Examples include some content service providers with common user IDs, while hospitals and pharmacies may share some common patient identities.

Before delving further, we define few terms that we use in this paper:

asynchronous partitions are vertical partitions of a dataset which are more generalised cases of synchronous partitions. Asynchronous partitions do not necessarily exhibit a coherent sequence of data between the partitions, for example, by having missing and duplicate instances, or not being indexed by the same identity column.

index set is a set, denoted by $I D$, of values for identities of all instances in a dataset or its partition.

Table 1. Synchronously (vertically) partitioned dataset

\begin{tabular}{|c|c|c|c|c|}
\hline \multicolumn{3}{|c|}{ Alice } & \multicolumn{2}{|c|}{ Bob } \\
\hline day & $\overline{A_{1}}$ & $\overline{A_{2}}$ & $A_{3}$ & $\bar{C}$ \\
\hline 1 & sunny & hot & high & no \\
\hline 2 & sunny & hot & low & yes \\
\hline 3 & rainy & hot & high & yes \\
\hline 4 & rainy & $\mathrm{cool}$ & low & yes \\
\hline
\end{tabular}

Table 2. Asynchronously partitioned dataset

\begin{tabular}{c|c|c}
\multicolumn{3}{c}{ Alice } \\
\hline ID & $A_{1}$ & $A_{2}$ \\
\hline 1 & sunny & hot \\
2 & sunny & hot \\
3 & rain & hot \\
4 & rain & cool \\
- & - & - \\
- & - & - \\
\hline
\end{tabular}

\begin{tabular}{c|c|c}
\multicolumn{3}{c}{ Bob } \\
\hline ID & $A_{3}$ & $C$ \\
\hline 1 & high & no \\
- & - & - \\
3 & low & yes \\
4 & low & yes \\
5 & high & yes \\
3 & high & yes \\
\hline
\end{tabular}


The simplest solution to the problem of asynchronously partitioned datasets is to sort instances by IDs so as to make the two datasets consistent by IDs, and then perform secure scalar product protocols on the vectors. However, it is not so easy. Attributes may be missing for certain IDs, e.g. for $i d=2$ in Bob's partition. One ID may have multiple instances, e.g. the $3 \mathrm{rd}$ and the 6 th instances conflict for the common $i d=3$ in Bob's partition. The most significant issue in asynchronous partitions is scalability. The conventional vector-based approaches are not efficient for datasets in which most instances are empty, i.e. sparse datasets and this leads us to what is often called the sparsity problem, e.g. [5].

missing values. Datasets may consist of missing values for some IDs, which contributes to low density of data. For example, the density is only 0.03 in "EachMovie" dataset2 [6]

duplicate assignment. Datasets may assign the same ID to distinct instances. The arbitrary assignment of IDs can make datasets inconsistent.

scalability. Vector-based approach requires processing for each element of the input vector even if most elements are empty. Such schemes do not scale well as the computational costs increase dramatically with increases in dataset size in sparse datasets.

Our goal and approaches: The goal of this paper is to construct a privacypreserving protocol for applying the Naïve Bayes classifier to vertically and asynchronously partitioned datasets, which deals with the issues of (1) missing values, (2) duplicate assignments, and (3) scalablity.

In order to address these issues in asynchronous partitions, we introduce the secure set intersection scheme presented by Agrawal et al. in [7, which uses commutative public-key encryption. The particular advantage of the scheme is that it works only on non-zero elements and is, therefore, appropriate for sparse datasets.

The contributions of this paper are: (1) first work of its kind, to our knowledge, to consider asynchronously partitioned datasets; (2) a secure privacy preserving scheme which scales well with the size of data and works efficiently with sparse datasets; (3) a performance based evaluation of an experimental implementation of the proposed scheme; and (4) an analytical evaluation of the scheme in terms of the how much information is revealed.

Organisation: This paper is organised as follows. In Section 2, we review some of the fundamental concepts and the existing work in privacy-preserving data mining. In section 3, we present our proposed scheme. In Section 4, we evaluate our scheme based on an experimental implementation.

\section{Building Blocks}

\subsection{Naïve Bayes Classifier}

Naïve Bayes is a widely used classifier based on the Bayes theorem, where the class with the highest likelihood is chosen. Since the algorithm is simple but efficient to implement, it is widely used for many purposes including email spam filtering, prediction of credit scoring, and so on.

\footnotetext{
${ }^{2}$ EachMovie dataset in the Grouplens project: http://www.grouplens.org/node/76
} 


\subsection{Secure Scalar Product Based Scheme}

Vaidya and Clifton proposed a privacy-preserving scheme for the Naïve Bayes classifier in 1. The method allows two parties, each having access to only one partition of a vertically partitioned dataset to predict the most likely target class for any given instance without revealing data from each other's partitions.

Algorithm 1. Secure Scalar Product

Input: Alice has $n$-dimentional vector $\boldsymbol{x}=\left(x_{1}, \ldots, x_{n}\right)$. Bob has $n$-dimentional $\boldsymbol{y}=$ $\left(y_{1}, \ldots, y_{n}\right)$.

Output: Alice has $s_{A}$ and Bob has $s_{B}$ such that $s_{A}+s_{B}=\boldsymbol{x} \cdot \boldsymbol{y}$.

1. Alice generates a homomorphic public-key pair and sends public key to Bob.

2. Alice sends to Bob $n$ ciphertexts $E\left(x_{1}\right), \ldots, E\left(x_{n}\right)$.

3. Bob chooses $s_{B}$ at random, computes

$$
c=E\left(x_{1}\right)^{y_{1}} \cdots E\left(x_{n}\right)^{y_{n}} / E\left(s_{B}\right)
$$

and send $c$ to Alice.

4. Alice decrypts $c$ to get $s_{A}=D(c)=x_{1} y_{1}+\cdots+x_{n} y_{n}-s_{B}$.

The drawback of the protocol is the strong assumption of a synchronous partition, i.e. (1) vectors $\boldsymbol{a}$ and $\boldsymbol{c}$ have the same dimension, (2) elements correspond to each other for two vectors.

The secure scalar product based methods, hence, can not simply be applied to asynchronously partitioned datasets such as Table 2.

\section{Proposed Scheme}

\section{$3.1 \quad$ Idea}

In order to perform Naïve Bayes in a scalable way, we introduce a secure set intersection protocol, which allows Alice and Bob with subsets $X$ and $Y$, respectively, to compute $X \cap Y$ without revealing $X$ or $Y$. Intersection is an useful primitive for many data mining algorithms and hence has been studied so far in 899. The scheme presented in [8] uses oblivious polynomial evaluation that suffers from the linear relation between computational cost and the order of the polynomial. It is, therefore, not appropriate for our purpose. For our study, we focus on the scheme presented by Agrawal, et. al. in [7, which uses commutative public-key encryption, which is performed only for active (i.e. not missing) elements and therefore is more appropriate for sparse datasets.

The aforementioned intersection protocol, however, reveals intermediate results to get the final prediction for the class variable, because one party must learn how many elements belong to both Alice and Bob in order to proceed with the protocol. On the other hand, the existing secure scalar product preserves the secrecy about the size of intersection $|X \cap Y|$ through an additional random number $\left(s_{B}\right.$ at Step 3 in Algorithm 1). The revealed information is critical to privacy preservation. Therefore, we propose a new secure protocol based on [7] in order to improve both privacy and scalability for privacy-preserving data mining. 


\subsection{Secure Set Intersection Protocol}

Agrawal, et. al. proposed, in [7], a secure intersection protocol using a publickey encryption algorithm that is commutative, i.e. $f(g(x))=g(f(x))$ and proved its security under assumption of semi-honest model and random-oracle model. For concrete discussion, we illustrate the scheme in Algorithm 2 using a power function $f_{e}(x)=x^{e} \bmod p$ defined under Decisional Diffie-Hellman hypothesis as commutative encryption 3 .

\section{Algorithm 2. Secure Intersection Protocol}

Input: Alice has subset $X=\left\{x_{1}, \ldots, x_{n_{A}}\right\}$, Bob has subset $Y=\left\{y_{1}, \ldots, y_{n_{B}}\right\}$.

Output: Intersection $|X \cap Y|$.

Let $Z_{q}$ be a multiplicative group with prime order $q$ and $H$ be a secure hash function that maps into range $G$.

1. Alice chooses random $u \in Z_{q}$ and send to Bob $H\left(x_{1}\right)^{u}, \ldots, H\left(x_{n_{A}}\right)^{u}$ in random order.

2. Bob chooses random $v \in Z_{q}$ and send to Alice $H\left(y_{1}\right)^{v}, \ldots, H\left(y_{n_{B}}\right)^{v}$ and $\left(H\left(x_{1}\right)^{u}\right)^{v}, \ldots,\left(H\left(x_{n_{A}}\right)^{u}\right)^{v}$ as well.

3. Alice computes $\left(H\left(y_{1}\right)^{v}\right)^{u}, \ldots,\left(H\left(y_{n_{B}}\right)^{v}\right)^{u}$ and selects pairs $\left(x_{j}, y_{i}\right)$ such that $H\left(y_{i}\right)^{v u}=H\left(x_{j}\right)^{u v}$; the number of pairs being the size of intersection $=|X \cap Y|$.

Algorithm 2 with an input of set of $n$ elements requires $n$ hash value evaluation, $2 n$ modular exponentiations for each party 4 , and $n$-element set comparison, which runs in $n \log n$ time with any appropriate algorithm. So, total complexity is $O(n)+O(n \log n)=O(n \log n)$, but the most significant cost is that for modular exponentiation. Supposing $t_{e}$ be a processing time for exponentiations, the cost of $2 n$ exponentiations is $2 n t_{e}$.

While the polynomial interpolation based algorithm in [8], known as popular intersection protocol, requires $O(n \log \log n)$ modular exponentiations for oblivious polynomial evaluation, we will show, later in this paper, that the computational cost is considerably large and hence the commutative encryption is proper for large-scale data mining.

\subsection{Proposed Protocol: Distorted Intersection}

The goal of our protocol is to compute a conditional probability of $c \in C$ given $a \in A_{j}, \operatorname{Pr}(c \mid a)$, where party $A$ has the attribute $A_{j}$ and $B$ has the target class $C$. We denote as index sets, $X$ and $Y$, defined over the ranges of $A_{j}$ and $C$, as

$$
X_{a, A_{j}}=\left\{i d \in I D \mid A_{j}(i d)=a\right\}, Y_{c}=\{i d \in I D \mid C(i d)=c\} .
$$

${ }^{3}$ For easy understanding, we describe a simplified protocol where only Alice learns the results.

${ }^{4} n=\max \left(n_{A}, n_{B}\right)+\epsilon$ where $\epsilon$ is a positive integral constant; thus $n$ is bigger than both $n_{A}$ and $n_{B}$. 
For instance, the datasets in Table 2 define the corresponding index sets for $a=$ sunny and $c=$ yes as $X_{\text {sunny, } A_{1}}=\{1,2\}$ and $Y_{\text {yes }}=\{3,4,5\}$ respectively.

In order to hide the size of intersection from Alice $(A)$, Bob $(B)$ wishes to add random noise to his secret input. However, $B$ does not know which elements belong to the intersection prior to the execution of the protocol. Hence, he makes his own input distorted by discarding some elements with random probability $p=s_{B} / n_{B}$ so that $A$ cannot learn the exact size of the intersection without knowledge of the random probability distribution.

With the randomisation step, the resulting size of the intersection is skewed with $p$ as $s_{A}=|X \cap Y| s_{B} / n_{B}$, which is known to $A$ who does not know $p$; while, $B$ knows $p$ but does not know $s_{A}$. Therefore, both parties participate in Yao's secure multi-party protocol to compute the multiplication

$$
s_{A} \cdot \frac{n_{B}}{s_{B}}=|X \cap Y|,
$$

which gives the conditional probability

$$
\operatorname{Pr}(X \mid Y)=\frac{|X \cap Y|}{|C|} .
$$

Finally, the prediction of target class for a given instance, $c_{N B}$, is obtained from Naïve Bayes classifier. Yao's protocol allows them to compare several candidates of the class without revealing any partial intermediate information.

Our proposed protocol is described in Algorithm 3 .

\section{Evaluation}

\subsection{Performance evaluation}

In order to evaluate performance improvement of the proposed scheme in comparison with others, we implement the following schemes for the secure Naïve Bayes classifier:

1. Scalar product based scheme, Vaidya and Clifton [1, which requires a homomorphic encryption and a secure function evaluation of comparison of additively shared value $\left(S F E_{1}\right)$,

2. Set intersection schemes, proposed by Freedman, Nissim and Pinkas [8, requiring a secure polynomial evaluation, and

3. Commutative encryption scheme, proposed in this paper, which requires a homomorphic encryption and a secure function evaluation of comparison of multiplicatively shared value $\left(S F E_{2}\right)$.

Test implementation. Our trial experimental system is implemented using Java (SDK 1.6.0) running on Intel Core2 Duo CPU 2.53 GHz, 2GB, Windows 7 (32 bit). We use the Paillier encryption with $\left|n^{2}\right|=2048$ bit modulus for additive homomorphic property, with a proprietary public key format. Our implementation has the average processing time of our trial implementation for encryption, decryption and modular exponentiation, denoted by $t_{E}=1.1, t_{D}=1.6$ and $t_{P}=0.15$, respectively. Note that the cost of decryption is higher than that of encryption because of property of Paillier encryption [10]. 
Algorithm 3. Distorted Intersection

Input: Alice has subset $X=\left\{x_{1}, \ldots, x_{n_{A}}\right\}$, Bob has subset $Y=\left\{y_{1}, \ldots, y_{n_{B}}\right\}$.

Output: shares of intersection, such that $s_{A} \cdot s_{B}=|X \cap Y|$.

1. Alice chooses random $u \in Z_{q}$, computes $H\left(x_{1}\right)^{u}, \ldots, H\left(x_{n_{A}}\right)^{u}$ and send to Bob in random order. Alternatively, she can sort these values in numerical order.

2. Bob chooses random $v \in Z_{q}$, computes $H\left(x_{1}\right)^{u v}, \ldots, H\left(x_{n_{A}}\right)^{u v}$ and send to Bob in random order.

3. Bob chooses random $s_{B}\left(<n_{B}\right)$ and for $i=1, \ldots, n_{B}$, compute

$$
w_{i}= \begin{cases}H\left(y_{i}\right)^{v} & \text { with probability }=s_{B} / n_{B}, \\ r_{i} & \text { otherwise }\end{cases}
$$

where $r_{i}$ is randomly chosen from $Z_{q}$ except $H\left(y_{i}\right)^{v}$ for every $i$. Then Bob sends $w_{1}, \ldots, w_{n_{B}}$ in random order to Alice.

4. Alice finds pairs $x_{j}, y_{i}$ such that $H\left(y_{i}\right)^{v u}=H\left(x_{j}\right)^{u v}$, and where $s_{A}$ is the number of pairs, i.e. $s_{A}\left(=|X \cap Y|\left(s_{B} / n_{B}\right)\right)$.

Secure Scalar Product. The secure scalar product based scheme requires $N$ encryptions and one decryption plus secure function evaluation of comparison of shared sum $\left(S F E_{1}\right)$, i.e.

$$
T_{1}=N t_{E}+t_{D}+S F E_{1}
$$

where $N$ is the dimension of the vectors. Note that mostly $N \gg n$, where $n$ is the number of active IDs in the asynchronously partitioned dataset.

Secure Function Evaluation (SFE). We use the generic two-party secure function evaluation evaluation system, Fairplay[11. Fairplay consists of a compiler of a high level procedural definition language, SFDL, into a one-pass Boolean circuit in a language called SHDL.

With Fairplay, we can perform secure function without revealing inputs. Figure 1 is the source code 'SharedCmp' to securely test $s_{A 0}+s_{B 0}>s_{A 1}+s_{B 1}$ where $s_{A 0}, s_{A 1}$ are owned by Alice and values $s_{0}$ and $s_{1}$, additively shared as $s_{0}=s_{A 0}+s_{B 0}$ are compared. The bit size is 16 .

The example shows that Fairplay allows us to code arbitrary functions easily. However, due to the processing cost, multiplication and division are not provided as primitive operations 11. We have to code those as programmed functions to perform comparison for multiplicatively shared values as $s_{A 0} \cdot s_{B 0}>s_{A 1} \cdot s_{B 1}$. (In our trial implementation, we omit the division since we can replace it by multiplication with some constant).

Table 3 shows the average processing time measured by Alice and Bob for several classes. Both parties have almost the same overhead to jointly evaluate comparison. In this experiment, we use 16-bit integers. 


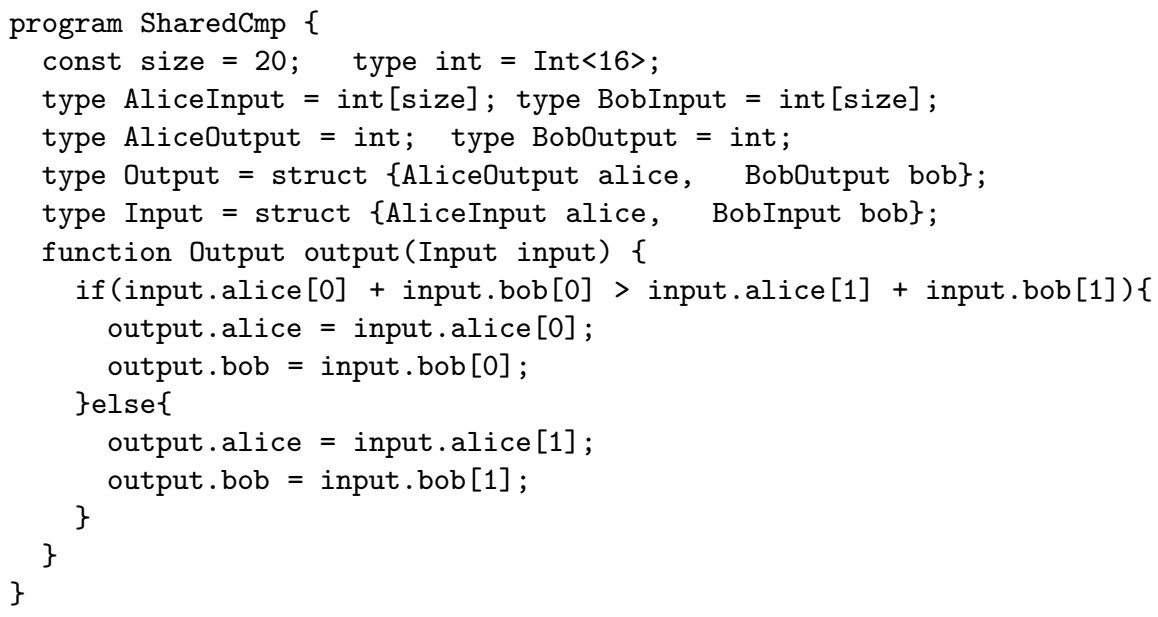

Fig. 1. Fairplay program (in SFDL) 'SharedCmp': shared integer comparison $s_{A 0}+$ $s_{B 0}>s_{A 1}+s_{B 1}$

Table 4 gives our estimation of performance for $S F E_{1}$ (addition) and $S F E_{2}$ (multiplication) based on the experimental measurement. Based on the runtime complexity, the curve fitting polynomial in terms of size of input $x$ and the processing time when $x=10 b i t s(=1024)$ are given. Figure 2 illustrates the estimation. We observe that the cost for secure multiplication increases with respect to the input size, and hence the our proposed scheme has a considerable large constant time overhead.

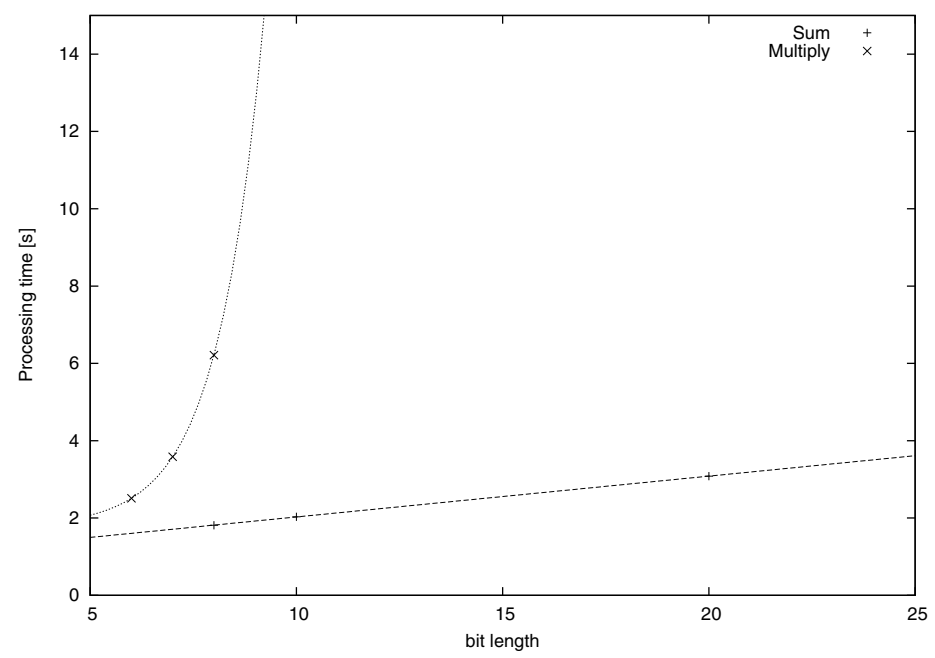

Fig. 2. Processing Time in Yao's SFE (Secure Function Evaluation) for sum and multiplication 
Table 3. Processing Time $S F E_{1}$ (Shared Comparison)

\begin{tabular}{l|r|r}
\hline & Alice & Bob \\
\hline Mean processing time (sec) & 0.80 & 0.82 \\
\hline
\end{tabular}

Table 4. Processing Time for $S F E_{1}$ (addition) and $S F E_{2}$ (multiplication) with size of 10 bit (=1024) interger

\begin{tabular}{r|c|r}
\hline circuit & fitting & Time (sec) \\
\hline$S F E_{1}$ (addition) & $0.97+0.106 x$ & 2.03 \\
$S F E_{2}$ (multiplication) & $1.77+0.003 e^{0.89 x}$ & 23.76 \\
\hline
\end{tabular}

Scalability of Proposed Scheme. Our proposed Algorithm 3 requires as many encryptions as the number of active users, $n$, and runs in time

$$
T_{2}=2 t_{P} n+t_{c} n \log n+S F E_{2}
$$

where $t_{c}$ is the cost of comparison of $n$ size lists. We may omit the overhead for comparison because $t_{c} \ll t_{e}, t_{d}$. Since $n \ll N$, it runs faster than the secure scalar product based protocol $\left(T_{1}\right)$. However, the constant overhead for secure function evaluation of multiplicatively shared values $\left(S F E_{2}\right)$ is higher than that of additive shared values $\left(S F E_{1}\right)$. The proposed protocol is scalable in terms of the entire size of dataset, $N$, but suffers the constant overhead of SFE.

Therefore, we conclude that the proposed scheme is efficient only for large sparse datasets such that

$$
N^{*} \geq \frac{S F E_{2}-S F E_{1}-t_{D}}{t_{E}-2 t_{P} \alpha},
$$

where $\alpha=n / N$ assuming $n$ is proportional to the entire size of dataset. We illustrate the scalability of our proposed scheme in Figure 3, where the proposed one is more efficient than the secure scalar product based scheme [1] when $N$ is large. Most of asynchronously partitioned datasets are considered as ones with small fractions of intersection. Consequently, we can say that the proposed scheme improves the performance for large scale sparse datasets.

\subsection{Security}

In [7], assuming the random oracle model and no hash collisions, and in semihonest model, there is no polynomial-time algorithm that can distinguish between a random value and $H(x)^{u}$ given $x$. This means that Algorithm 2 preserves the privacy of input subsets $X$ and $Y$. With zero-knowledge proof, the security in the random oracle model can even be extended to a malicious model where parties behave arbitrarily.

Algorithm 1 is also proved as secure even after one party (Alice) learns the result of the protocol, $s_{A}=x_{1} y_{1}+\cdots+x_{n} y_{n}-s_{B}$, which is randomised with 


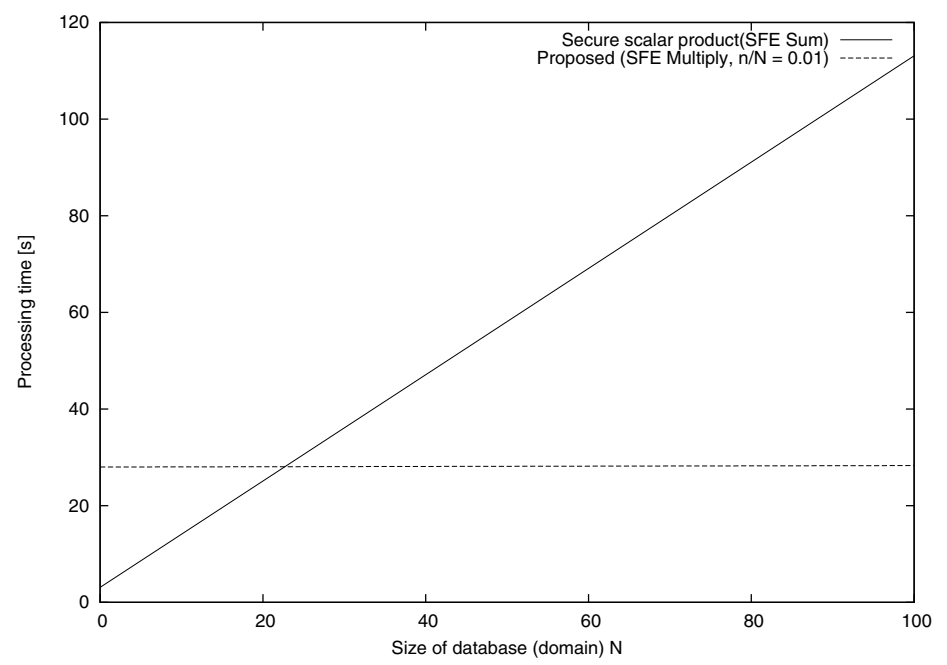

Fig. 3. Scalability in terms of processing time for the size of database (set of indexes) $N$

$s_{B}$ chosen uniformly by the other party (Bob). More formally, learning partial result $s_{A}$ reveals nothing about the distribution of $s_{A}+s_{B}$, which is distributed uniformly over group $Z_{q}$ of order $q$, that is, the conditional probability of the sum given $s_{A}$ is identical to apriori probability, i.e. $\operatorname{Pr}\left(s_{A}+s_{B} \mid s_{A}\right)=\operatorname{Pr}\left(s_{A}+s_{B}\right)=$ $1 / q$. SFE also preserves the secrecy of shared inputs under the assumptions of semantically secure public key algorithm.

However, the distortion in Algorithm 3 is not uniform. Let $z$ be the size of intersection $|X \cap Y|$, and $p$ be a probability to apply commutative encryption in the algorithm, defined as $p=s_{B} / n_{B}$. The conditional probability of the algorithm outputs $s_{A}$ given $z$ is computed with the binomial distribution as:

$$
\operatorname{Pr}\left(s_{A} \mid z\right)= \begin{cases}0 & \text { if } s_{A}>z \\
\left(\begin{array}{c}
z \\
s_{A}
\end{array}\right) p^{s_{A}}(1-p)^{z-s_{A}} & \text { otherwise. }\end{cases}
$$

Then, what can Alice guess about $z$ after she learns the output of the algorithm, $s_{A}$ ?

Table 5. Summary of proposed scheme

\begin{tabular}{|c|c|c|}
\hline $\begin{array}{l}\text { scheme: } \\
\text { based on: }\end{array}$ & $\begin{array}{c}\text { Vaidya \& Clifton }[1] \\
\text { Secure Scalar Product }[12]\end{array}$ & $\begin{array}{c}\text { Proposed } \\
\text { Commutative encryption [7] }\end{array}$ \\
\hline $\begin{array}{c}\text { input } \\
\text { computation cost } \\
\text { accuracy } \\
\text { security }\end{array}$ & $\begin{array}{c}N \text {-dimension binary vectors } \\
T_{1}=t_{E} N+t_{D}+S F E_{1} \\
\text { accurate } \\
\operatorname{Pr}(z \mid X . Y)=1 / N\end{array}$ & $\begin{array}{c}\text { integer subset of size } n \\
T_{3}=t_{P} n+n \log n+S F E_{2} \\
\text { accurate with probability } s_{B} / n_{B} \\
\operatorname{Pr}\left(z \mid s_{A}\right)>1 / n\end{array}$ \\
\hline
\end{tabular}


Bayes theorem gives to her an useful hint, i.e. the probability distribution of $z$ as

$$
\operatorname{Pr}\left(z \mid s_{A}\right)=\frac{\operatorname{Pr}\left(s_{A} \mid z\right) \operatorname{Pr}(z)}{\operatorname{Pr}\left(s_{A}\right)}=\frac{\left.\operatorname{Pr}\left(s_{A} \mid z\right) 1 /(n+1)\right)}{\sum_{z} \operatorname{Pr}\left(s_{A} \mid z\right) \operatorname{Pr}(z)},
$$

where we assumes $\operatorname{Pr}(z)=1 /(n+1)$.

\section{Conclusion}

We have proposed a scalable privacy-preserving Naïve Bayes classifier for asynchronously partitioned datasets. Our proposed scheme is based on the work presented in [7] using a public-key encryption algorithm that satisfies commutative property. The performance of our proposed protocol is shown to be better than the scheme based on the secure scalar product [1] when the matrix is sparse, i.e. most entries are missing and the fraction of active data is small, that is $n \ll N$, which frequently happens in asynchronously partitioned datasets. Table 5 gives the summary of the features of our proposed protocol.

\section{References}

1. Vaidya, J., Clifton, C.: Privacy Preserving Naïve Bayes Classifier for Vertically Partitioned Data. In: SIAM International Conference on Data Mining, Lake Buena Vista, Florida, pp. 522-526. Society of Industrial and Applied Mathematics, Philadelphia (2004)

2. Douceur, J.: The sybil attack. In: Druschel, P., Kaashoek, M.F., Rowstron, A. (eds.) IPTPS 2002. LNCS, vol. 2429, pp. 251-260. Springer, Heidelberg (2002)

3. Yu, H., Shi, C., Kaminsky, M., Gibbons, P.B., Xiao, F.: DSybil: Optimal Sybilresistance for Recommendation Systems. In: 30th IEEE Symposium on Security and Privacy, pp. 283-298. IEEE, Los Alamitos (2009)

4. Yao, A.C.C.: How to generate and exchange secrets. In: 27th Annual Symposium on Foundations of Computer Science, pp. 162-167. IEEE, Los Alamitos (1986)

5. Zhou, J., Luo, T.: A novel approach to solve the sparsity problem in collaborative filtering. In: International Conference on Networking, Sensing and Control (ICNSC), pp. 165-170. IEEE, Los Alamitos (2010)

6. GroupLens: GroupLens Research, http://www.grouplens.org/ (2010)

7. Agrawal, R., Evfimievski, A., Srikant, R.: Information sharing across private databases. In: The ACM SIGMOD International Conference on Management of Data, pp. 86-97. ACM, New York (2003)

8. Freedman, M.J., Nissim, K., Pinkas, B.: Efficient private matching and set intersection. In: Cachin, C., Camenisch, J.L. (eds.) EUROCRYPT 2004. LNCS, vol. 3027, pp. 1-19. Springer, Heidelberg (2004)

9. Vaidya, J., Clifton, C.: Secure set intersection cardinality with application to association rule mining. Journal of Computer Security 13(4), 593-622 (2005)

10. Paillier, P.: Public-key cryptosystems based on composite degree residuosity classes. In: Stern, J. (ed.) EUROCRYPT 1999. LNCS, vol. 1592, pp. 223-238. Springer, Heidelberg (1999)

11. Malkhi, D., Nisan, N., Pinkas, B., Sella, Y.: Fairplay - a secure two-party computation system. In: The 13th USENIX Conference on Security Symposium, p. 20. USENIX Association (2004) 
12. Du, W., Atallah, M.J.: Privacy-preserving cooperative statistical analysis. In: 17th Annual Computer Security Applications Conference, ACSAC, pp. 102-110. IEEE, Los Alamitos (2001)

13. Kikuchi, H., Kizawa, H., Tada, M.: Privacy-Preserving Collaborative Filtering Schemes. In: International Conference on Availability, Reliability and Security, ARES 2009, pp. 911-916. IEEE, Los Alamitos (2009)

14. Sarwar, B., Karypis, G., Konstan, J., Reidl, J.: Item-based collaborative filtering recommendation algorithms. In: The 10th International Conference on World Wide Web, pp. 285-295. ACM, New York (2001) 